\title{
Some Extensions on Cerone's Generalizations of Steffensen's Inequality
}

\section{${ }^{1}$ Atta Abu Hany and ${ }^{2}$ Mahmoud Al Agha}

${ }^{1}$ Dep. Of Mathematics, Alazhar Univ. of Gaza, Palestine, attahany@gmai1. com

${ }^{2}$ Dep. Of Mathematics, Alazhar Univ. of Gaza, Palestine, m. elagha89@hotmail. com

Abstract This paper provides more extensions on Cerone's generalizations of Steffensen's inequality with bounds involving any two subintervals. Moreover, we introduce some applications for integral mean.

Keywords. Steffensen inequality, monotonic functions, integral mean.

2010 Mathematics Subject Classification : 26 D 15, 26 D 10 .

\section{Introduction}

Inequalities are at the heart of mathematical analysis [3, 4]. Since its appearance in 1918, Steffensen's inequality has been applied to a wide range of topics in mathematics and statistics (see $[5,6])$. It lies in the core of integral inequalities, which can be used for dealing with the comparison between integrals over a whole interval $[a, b]$ and integrals over a subinterval of $[a, b]$. The following is the original Steffensen's inequality [6] .

Theorem 1.1 [6 ] Assume that two integrable functions $f(t)$ and $g(t)$ are defined on the interval $[a, b]$ with $f(t)$ non-increasing and that $0 \leq g(t) \leq 1$ on $[a, b]$. Then

$$
\int_{b-\lambda}^{b} f(t) d t \leq \int_{a}^{b} f(t) g(t) d t \leq \int_{a}^{a+\lambda} f(t) d t
$$

where

$$
\lambda=\int_{a}^{b} g(t) d t
$$

The inequalities are reversed for $f$ non-decreasing.

Steffensen's inequality has been generalized in many ways. In [ 2], the authors extended Cerone's generalization of Steffensen's inequality to positive finite measures and to give weaker conditions for obtained extension. In the following section we present some of these generalizations given by Cerone [1].

\section{Extensions of Cerone's Results}

The following lemma will be useful for the results that follow.

Lemma 2.1[1] Let $f, g:[a, b] \rightarrow \mathbf{R}$ be integrable functions on $[a, b]$. Further, let $[c, d] \subseteq[a, b]$ with $\lambda=d-c=\int_{a}^{b} g(t) d t$. Then the following identities hold. Namely, 


$$
\begin{aligned}
\int_{c}^{d} f(t) d t-\int_{a}^{b} f(t) g(t) d t=\int_{a}^{c} & (f(d)-f(t)) g(t) d t+\int_{c}^{d}(f(t)-f(d))(1-g(t)) d t \\
& +\int_{d}^{b}(f(d)-f(t)) g(t) d t
\end{aligned}
$$

and

$$
\begin{aligned}
\int_{a}^{b} f(t) g(t) d t-\int_{c}^{d} f(t) d t=\int_{a}^{c} & (f(t)-f(c)) g(t) d t+\int_{c}^{d}(f(c)-f(t))(1-g(t)) d t \\
& +\int_{d}^{b}(f(t)-f(c)) g(t) d t .
\end{aligned}
$$

In his excellent paper [1], Cerone provides a generalization of Steffensen's inequality which allows bounds involving any two subintervals instead of restricting them to include the end points.

Theorem 2.1 [1] Let $f, g:[a, b] \rightarrow \mathbf{R}$ be integrable functions on $[a, b]$ and let $f$ be nonincreasing. Further, let $0 \leq g(t) \leq 1$ and $\lambda=\int_{a}^{b} g(t) d t=d_{i}-c_{i}$, where $\left[c_{i}, d_{i}\right] \subset[a, b]$ for $i=1,2$ and $d_{1} \leq d_{2}$.

(a) Then

$$
\int_{a}^{b} f(t) g(t) d t \leq \int_{c_{1}}^{d_{1}} f(t) d t+R\left(c_{1}, d_{1}\right)
$$

holds where,

$$
R\left(c_{1}, d_{1}\right)=\int_{a}^{c_{1}}\left(f(t)-f\left(d_{1}\right)\right) g(t) d t \geq 0 .
$$

(b) Then

$$
\int_{c_{2}}^{d_{2}} f(t) d t-r\left(c_{2}, d_{2}\right) \leq \int_{a}^{b} f(t) g(t) d t
$$

holds where,

$$
r\left(c_{2}, d_{2}\right)=\int_{d_{2}}^{b}\left(f\left(c_{2}\right)-f(t)\right) g(t) d t \geq 0
$$

Remark 2.1 If in Theorem 2.1 we take $c_{1}=a$ and so $d_{1}=a+\lambda$, then $R(a, a+\lambda)=0$. Further, taking $d_{2}=b$ so that $c_{2}=b-\lambda$, gives $r(b-\lambda, b)=0$. The Steffensen's inequality (1.1) is thus recaptured. Since (1.2) holds, then $c_{2} \geq a$ and $d_{1} \leq b$ giving $\left[c_{i}, d_{i}\right] \subset[a, b]$. Theorem 2.1 may thus be viewed as a generalization of the Steffensen's inequality as given in Theorem 1.1, to allow for two equal length subintervals that are not necessarily at the ends of $[a, b]$.

Corollary 2.1[1] Let the conditions of Theorem 2.1 hold.

(a) Then

$$
\int_{a}^{b} f(t) g(t) d t \leq \int_{a}^{d_{1}} f(t) d t-\left(c_{1}-a\right) f\left(d_{1}\right)
$$

(b) Then

$$
\int_{c_{2}}^{b} f(t) d t-\left(b-d_{2}\right) f\left(c_{2}\right) \leq \int_{a}^{b} f(t) g(t) d t
$$

Remark 2.2 If we take $c_{1}=a$ and so $d_{1}=a+\lambda$ and $d_{2}=b$ such that $c_{2}=b-\lambda$ then (2.7) and (2.8) together again recaptures Steffensen's inequality as given in Theorem 1.1.

The following theorem is an extension to Theorem 2.1 for the case $i=1,2, \ldots, n$. 
Theorem 2.2 Let $f, g:[a, b] \rightarrow \mathbf{R}$ be integrable functions on $[a, b]$ and let $f$ be negative and nonincreasing. Further, let $0 \leq g(t) \leq 1$ and $\lambda=\int_{a}^{b} g(t) d t=d_{i}-c_{i}$, where $\left[c_{i}, d_{i}\right] \subset[a, b]$ for $i=1,2, \ldots, n$ and $d_{1} \leq d_{2} \leq \ldots \leq d_{n}, n=2,3,4, \ldots$.

(a) Then

$$
\begin{gathered}
(n-1) \int_{a}^{b} f(t) g(t) d t \leq \int_{c_{1}}^{d_{1}} f(t) d t+\int_{c_{2}}^{d_{2}} f(t) d t+\ldots+\int_{c_{n-1}}^{d_{n-1}} f(t) d t \\
+R\left(c_{n-1}, d_{n-1}\right)
\end{gathered}
$$

holds where,

$$
\begin{aligned}
R\left(c_{n-1}, d_{n-1}\right)= & \int_{a}^{c_{1}}\left(f(t)-f\left(d_{1}\right)\right) g(t) d t+\int_{a}^{c_{2}}\left(f(t)-f\left(d_{2}\right)\right) g(t) d t+\ldots+ \\
& +\int_{a}^{c_{n-1}}\left(f(t)-f\left(d_{n-1}\right)\right) g(t) d t \geq 0 .
\end{aligned}
$$

(b) Then

$$
\begin{aligned}
& \int_{c_{2}}^{d_{2}} f(t) d t+\int_{c_{3}}^{d_{3}} f(t) d t+\ldots+\int_{c_{n-1}}^{d_{n-1}} f(t) d t+\int_{c_{n}}^{d_{n}} f(t) d t-r\left(c_{n}, d_{n}\right) \\
& \leq(n-1) \int_{a}^{b} f(t) g(t) d t
\end{aligned}
$$

holds where,

$$
\begin{gathered}
r\left(c_{n}, d_{n}\right)=\int_{d_{2}}^{b}\left(f\left(c_{2}\right)-f(t)\right) g(t) d t+\int_{d_{3}}^{b}\left(f\left(c_{3}\right)-f(t)\right) g(t) d t+\ldots+ \\
+\int_{d_{n-1}}^{b}\left(f\left(c_{n-1}\right)-f(t)\right) g(t) d t+\int_{d_{n}}^{b}\left(f\left(c_{n}\right)-f(t)\right) g(t) d t \geq 0 .
\end{gathered}
$$

Proof of part (a). Since $f$ is non-increasing on $\left[c_{1}, d_{1}\right]$ for $t \leq d_{1}$, on $\left[c_{2}, d_{2}\right]$ for $t \leq d_{2}$, $\ldots$, on $\left[c_{n-1}, d_{n-1}\right]$ for $t \leq d_{n-1}$ respectively, then $f(t) \geq f\left(d_{1}\right), f(t) \geq f\left(d_{2}\right), \ldots$, $f(t) \geq f\left(d_{n-1}\right)$ respectively. Similarly, $f$ is non-increasing on $\left[d_{1}, b\right]$ for $t \geq d_{1}$, on $\left[d_{2}, b\right]$ for $t \geq d_{2}, \ldots$, on $\left[d_{n-1}, b\right]$ for $t \geq d_{n-1}$, respectively, then $f(t) \leq f\left(d_{1}\right), f(t) \leq f\left(d_{2}\right)$, $\ldots, f(t) \leq f\left(d_{n-1}\right)$ respectively.

Also since $0 \leq g(t) \leq 1$, we get $0 \geq-g(t) \geq-1$, then $0 \leq 1-g(t) \leq 1$, and from Lemma 2.1 , we obtain

$$
\begin{aligned}
& S\left(c_{1}, d_{1} ; a, b\right)+S\left(c_{2}, d_{2} ; a, b\right)+\ldots+S\left(c_{n-1}, d_{n-1} ; a, b\right)+\int_{a}^{c_{1}}\left(f(t)-f\left(d_{1}\right)\right) g(t) d t \\
& \quad+\int_{a}^{c_{2}}\left(f(t)-f\left(d_{2}\right)\right) g(t) d t+\ldots+\int_{a}^{c_{n-1}}\left(f(t)-f\left(d_{n-1}\right)\right) g(t) d t \\
& =\int_{c_{1}}^{d_{1}}\left(f(t)-f\left(d_{1}\right)\right)(1-g(t)) d t+\int_{c_{2}}^{d_{2}}\left(f(t)-f\left(d_{2}\right)\right)(1-g(t)) d t+\ldots+ \\
& \quad+\int_{c_{n-1}}^{d_{n-1}}\left(f(t)-f\left(d_{n-1}\right)\right)(1-g(t)) d t+\int_{d_{1}}^{b}\left(f\left(d_{1}\right)-f(t)\right) g(t) d t \\
& \quad+\int_{d_{2}}^{b}\left(f\left(d_{2}\right)-f(t)\right) g(t) d t+\ldots+\int_{d_{n-1}}^{b}\left(f\left(d_{n-1}\right)-f(t)\right) g(t) d t \geq 0 .
\end{aligned}
$$

Hence

$$
\int_{c_{1}}^{d_{1}} f(t) d t+\int_{c_{2}}^{d_{2}} f(t) d t+\ldots+\int_{c_{n-1}}^{d_{n-1}} f(t) d t-\int_{a}^{b} f(t) g(t) d t
$$




$$
\begin{aligned}
& -\int_{a}^{b} f(t) g(t) d t-\ldots-\int_{a}^{b} f(t) g(t) d t+\int_{a}^{c_{1}}\left(f(t)-f\left(d_{1}\right)\right) g(t) d t \\
& +\int_{a}^{c_{2}}\left(f(t)-f\left(d_{2}\right)\right) g(t) d t+\ldots+\int_{a}^{c_{n-1}}\left(f(t)-f\left(d_{n-1}\right)\right) g(t) d t \geq 0
\end{aligned}
$$

and thus (2.9) is valid. The term $R\left(c_{n-1}, d_{n-1}\right)$ is nonnegative since $f$ is non-increasing and $g$ is nonnegative. The proof of part (a) is completed.

Proof of part (b). Since $f$ is non-increasing on $\left[a, c_{2}\right]$ for $t \leq c_{2}$, on $\left[a, c_{3}\right]$ for $t \leq c_{3}$, $\ldots$, on $\left[a, c_{n}\right]$ for $t \leq c_{n}$ respectively, then $f(t) \geq f\left(c_{2}\right), f(t) \geq f\left(c_{3}\right), \ldots$, $f(t) \geq f\left(c_{n}\right)$ respectively. Similarly, $f$ is non-increasing on $\left[c_{2}, d_{2}\right]$ for $t \geq c_{2}$, on $\left[c_{3}, d_{3}\right]$ for $t \geq c_{3}, \ldots$, on $\left[c_{n}, d_{n}\right]$ for $t \geq c_{n}$, respectively, then $f(t) \leq f\left(c_{2}\right), f(t) \leq f\left(c_{3}\right)$, $\ldots, f(t) \leq f\left(c_{n}\right)$ respectively.

Also since $0 \leq g(t) \leq 1$, we get $0 \geq-g(t) \geq-1$, then $0 \leq 1-g(t) \leq 1$, and from Lemma 2.1, we obtain

$$
\begin{aligned}
& -S\left(c_{2}, d_{2} ; a, b\right)-S\left(c_{3}, d_{3} ; a, b\right)-\ldots-S\left(c_{n}, d_{n} ; a, b\right)+\int_{d_{2}}^{b}\left(f\left(c_{2}\right)-f(t)\right) g(t) d t \\
& \quad+\int_{d_{3}}^{b}\left(f\left(c_{3}\right)-f(t)\right) g(t) d t+\ldots+\int_{d_{n}}^{b}\left(f\left(c_{n}\right)-f(t)\right) g(t) d t \\
& =\int_{a}^{c_{2}}\left(f(t)-f\left(c_{2}\right)\right) g(t) d t+\int_{a}^{c_{3}}\left(f(t)-f\left(c_{3}\right)\right) g(t) d t+\ldots \\
& \quad+\int_{a}^{c_{n}}\left(f(t)-f\left(c_{n}\right)\right) g(t) d t+\int_{c_{2}}^{d_{2}}\left(f\left(c_{2}\right)-f(t)\right)(1-g(t)) d t \\
& \quad+\int_{c_{3}}^{d_{3}}\left(f\left(c_{3}\right)-f(t)\right)(1-g(t)) d t+\ldots+\int_{c_{n}}^{d_{n}}\left(f\left(c_{n}\right)-f(t)\right)(1-g(t)) d t \geq 0 .
\end{aligned}
$$

Hence

$$
\begin{array}{rl}
\int_{a}^{b} & f(t) g(t) d t+\int_{a}^{b} f(t) g(t) d t+\ldots+\int_{a}^{b} f(t) g(t) d t-\int_{c_{2}}^{d_{2}} f(t) d t \\
& -\int_{c_{3}}^{d_{3}} f(t) d t-\ldots-\int_{c_{n}}^{d_{n}} f(t) d t+\int_{d_{2}}^{b}\left(f\left(c_{2}\right)-f(t)\right) g(t) d t \\
& +\int_{d_{3}}^{b}\left(f\left(c_{3}\right)-f(t)\right) g(t) d t+\ldots+\int_{d_{n}}^{b}\left(f\left(c_{n}\right)-f(t)\right) g(t) d t \geq 0
\end{array}
$$

giving (2.11). The term $r\left(c_{n}, d_{n}\right)$ is nonnegative since $f$ is non-increasing and $g$ is nonnegative. The proof of part (b) is completed.

The following lemma will be used in recapturing the classical Steffensen's inequality (1.1).

Lemma 2.2 Let $f, g:[a, b] \rightarrow \mathbf{R}$ be integrable functions on $[a, b]$ and let $f$ be negative and nonincreasing. Further, let $0 \leq g(t) \leq 1$ and $\lambda=\int_{a}^{b} g(t) d t$. Then

$$
\begin{aligned}
& (n-2) \int_{b-\lambda}^{b} f(t) d t+(n-2) \int_{a}^{b-\lambda}(f(t)-f(b)) g(t) d t \\
\leq & (n-2) \int_{a}^{a+\lambda} f(t) d t .
\end{aligned}
$$

Proof.

$$
\begin{aligned}
& (n-2) \int_{b-\lambda}^{b} f(t) d t+(n-2) \int_{a}^{b-\lambda}(f(t)-f(b)) g(t) d t \\
& \leq(n-2) \int_{b-\lambda}^{b} f(t) d t+(n-2) \int_{a}^{b-\lambda}(f(t)-f(b)) d t
\end{aligned}
$$




$$
\begin{aligned}
& =(n-2) \int_{b-\lambda}^{b} f(t) d t+(n-2) \int_{a}^{b-\lambda} f(t) d t-(n-2) f(b) \int_{a}^{b-\lambda} d t \\
& =(n-2) \int_{a}^{b} f(t) d t-(n-2) f(b)(b-\lambda-a) \\
& =(n-2) \int_{a}^{b} f(t) d t+(n-2) f(b)(a+\lambda-b) \\
& \left.\leq(n-2) \int_{a}^{b} f(t) d t+(n-2) f(b)(b-b) \quad \text { (where } a \leq a+\lambda \leq b\right) \\
& =(n-2) \int_{a}^{b} f(t) d t \\
& \leq(n-2) \int_{a}^{a+\lambda} f(t) d t
\end{aligned}
$$

where $a \leq a+\lambda \leq b$ and $f$ is negative and non-increasing.

Remark 2.3 If we let $c_{1}=a$ in Theorem 2.2, we obtain $d_{1}=a+\lambda$. Further, taking $d_{2}=d_{3}=\ldots=d_{n}=b$ implies $c_{2}=c_{3}=\ldots=c_{n}=b-\lambda$. Then (2.9) and (2.11) together becomes

$$
\begin{aligned}
& \int_{b-\lambda}^{b} f(t) d t+\int_{b-\lambda}^{b} f(t) d t+\ldots+\int_{b-\lambda}^{b} f(t) d t \leq(n-1) \int_{a}^{b} f(t) g(t) d t \\
\leq & \int_{a}^{a+\lambda} f(t) d t+\int_{b-\lambda}^{b} f(t) d t+\ldots+\int_{b-\lambda}^{b} f(t) d t+\int_{a}^{b-\lambda}(f(t)-f(b)) g(t) d t+\ldots+ \\
& +\int_{a}^{b-\lambda}(f(t)-f(b)) g(t) d t .
\end{aligned}
$$

Hence

$$
\begin{aligned}
& (n-1) \int_{b-\lambda}^{b} f(t) d t \leq(n-1) \int_{a}^{b} f(t) g(t) d t \\
& \quad \leq \int_{a}^{a+\lambda} f(t) d t+(n-2) \int_{b-\lambda}^{b} f(t) d t+(n-2) \int_{a}^{b-\lambda}(f(t)-f(b)) g(t) d t .
\end{aligned}
$$

Using Lemma 2.2, we obtain

$$
(n-1) \int_{b-\lambda}^{b} f(t) d t \leq(n-1) \int_{a}^{b} f(t) g(t) d t \leq \int_{a}^{a+\lambda} f(t) d t+(n-2) \int_{a}^{a+\lambda} f(t) d t .
$$

The classical Steffensen's inequality (1.1) is thus recaptured.

Corollary 2.3 Let the conditions of Theorem 2.2 hold.

(a) Then

$$
\begin{aligned}
(n-1) \int_{a}^{b} f(t) g(t) d t & \leq \int_{a}^{d_{1}} f(t) d t+\int_{a}^{d_{2}} f(t) d t+\ldots+\int_{a}^{d_{n-1}} f(t) d t-\left(c_{1}-a\right) f\left(d_{1}\right) \\
& -\left(c_{2}-a\right) f\left(d_{2}\right)-\ldots-\left(c_{n-1}-a\right) f\left(d_{n-1}\right) .
\end{aligned}
$$

(b) Then

$$
\begin{gathered}
\int_{c_{2}}^{b} f(t) d t+\int_{c_{3}}^{b} f(t) d t+\ldots+\int_{c_{n}}^{b} f(t) d t-\left(b-d_{2}\right) f\left(c_{2}\right)-\left(b-d_{3}\right) f\left(c_{3}\right)-\ldots- \\
-\left(b-d_{n}\right) f\left(c_{n}\right) \leq(n-1) \int_{a}^{b} f(t) g(t) d t .
\end{gathered}
$$

Proof of part (a). From Theorem 2.2 and using $0 \leq g(t) \leq 1$, we obtain

$$
\begin{aligned}
0 \leq R\left(c_{n-1}, d_{n-1}\right) & =\int_{a}^{c_{1}}\left(f(t)-f\left(d_{1}\right)\right) g(t) d t+\ldots+\int_{a}^{c_{n-1}}\left(f(t)-f\left(d_{n-1}\right)\right) g(t) d t \\
\leq & \int_{a}^{c_{1}}\left(f(t)-f\left(d_{1}\right)\right) d t+\ldots+\int_{a}^{c_{n-1}}\left(f(t)-f\left(d_{n-1}\right)\right) d t \\
& =\int_{a}^{c_{1}} f(t) d t-\left(c_{1}-a\right) f\left(d_{1}\right)+\ldots+\int_{a}^{c_{n-1}} f(t) d t-\left(c_{n-1}-a\right) f\left(d_{n-1}\right),
\end{aligned}
$$

and so 


$$
\begin{aligned}
(n-1) \int_{a}^{b} f(t) g(t) d t \leq & \int_{c_{1}}^{d_{1}} f(t) d t+\ldots+\int_{c_{n-1}}^{d_{n-1}} f(t) d t+R\left(c_{n-1}, d_{n-1}\right) \\
\leq & \int_{c_{1}}^{d_{1}} f(t) d t+\ldots+\int_{c_{n-1}}^{d_{n-1}} f(t) d t+\int_{a}^{c_{1}} f(t) d t \\
& \quad-\left(c_{1}-a\right) f\left(d_{1}\right)+\ldots+\int_{a}^{c_{n-1}} f(t) d t-\left(c_{n-1}-a\right) f\left(d_{n-1}\right),
\end{aligned}
$$

giving the inequality (2.14).

Proof of part (b). From Theorem 2.2 and using $0 \leq g(t) \leq 1$, we obtain

$$
\begin{aligned}
0 \leq r\left(c_{n}, d_{n}\right) & =\int_{d_{2}}^{b}\left(f\left(c_{2}\right)-f(t)\right) g(t) d t+\ldots+\int_{d_{n}}^{b}\left(f\left(c_{n}\right)-f(t)\right) g(t) d t \\
& \leq \int_{d_{2}}^{b}\left(f\left(c_{2}\right)-f(t)\right) d t+\ldots+\int_{d_{3}}^{b}\left(f\left(c_{3}\right)-f(t)\right) d t \\
& =\left(b-d_{2}\right) f\left(c_{2}\right)-\int_{d_{2}}^{b} f(t) d t+\ldots+\left(b-d_{n}\right) f\left(c_{n}\right)-\int_{d_{n}}^{b} f(t) d t,
\end{aligned}
$$

and so

$$
\begin{aligned}
(n-1) \int_{a}^{b} f(t) g(t) d t & \geq \int_{c_{2}}^{d_{2}} f(t) d t+\ldots+\int_{c_{n}}^{d_{n}} f(t) d t-r\left(c_{n}, d_{n}\right) \\
\geq & \int_{c_{2}}^{d_{2}} f(t) d t+\ldots+\int_{c_{n}}^{d_{n}} f(t) d t-\left(b-d_{2}\right) f\left(c_{2}\right) \\
& +\int_{d_{2}}^{b} f(t) d t-\ldots-\left(b-d_{n}\right) f\left(c_{n}\right)+\int_{d_{n}}^{b} f(t) d t .
\end{aligned}
$$

Thus, the inequality of (2.15) is valid.

The following lemma will be used in recapturing the classical Steffensen's inequality (1.1).

Lemma 2.3 Let $f, g:[a, b] \rightarrow \mathbf{R}$ be integrable functions on $[a, b]$ and let $f$ be negative and nonincreasing. Further, let $0 \leq g(t) \leq 1$ and $\lambda=\int_{a}^{b} g(t) d t$. Then

\section{Proof.}

$$
(n-2) \int_{a+\lambda}^{b} f(t) d t-(n-2)(b-\lambda-a) f(b) \leq 0 .
$$

$$
\begin{aligned}
& (n-2) \int_{a+\lambda}^{b} f(t) d t-(n-2)(b-\lambda-a) f(b) \\
= & (n-2) \int_{a+\lambda}^{b} f(t) d t+(n-2)(a+\lambda-b) f(b) \\
\leq & (n-2) \int_{a+\lambda}^{b} f(t) d t+(n-2)(b-b) f(b) \quad(\text { where } a \leq a+\lambda \leq b) \\
= & (n-2) \int_{a+\lambda}^{b} f(t) d t \leq 0,
\end{aligned}
$$

where $a \leq a+\lambda \leq b$ and $f$ is negative and non-increasing.

Remark 2.4 If we let $c_{1}=a$ in Corollary 2.2, we obtain $d_{1}=a+\lambda$. Further, taking $d_{2}=d_{3}=\ldots=d_{n}=b$ implies $c_{2}=c_{3}=\ldots=c_{n}=b-\lambda$. Then (2.14) and (2.15) together becomes $\int_{b-\lambda}^{b} f(t) d t+\ldots+\int_{b-\lambda}^{b} f(t) d t \leq(n-1) \int_{a}^{b} f(t) g(t) d t$ $\leq \int_{a}^{a+\lambda} f(t) d t+\int_{a}^{b} f(t) d t+\ldots+\int_{a}^{b} f(t) d t-(b-\lambda-a) f(b)-\ldots-(b-\lambda-a) f(b)$.

Then

$$
(n-1) \int_{b-\lambda}^{b} f(t) d t \leq(n-1) \int_{a}^{b} f(t) g(t) d t
$$




$$
\leq \int_{a}^{a+\lambda} f(t) d t+(n-2) \int_{a}^{b} f(t) d t-(n-2)(b-\lambda-a) f(b) .
$$

Hence

$$
\begin{aligned}
& (n-1) \int_{b-\lambda}^{b} f(t) d t \leq(n-1) \int_{a}^{b} f(t) g(t) d t \\
& \quad \leq(n-2+1) \int_{a}^{a+\lambda} f(t) d t+(n-2) \int_{a+\lambda}^{b} f(t) d t-(n-2)(b-\lambda-a) f(b) .
\end{aligned}
$$

Using Lemma 2.3, we obtain

$$
(n-1) \int_{b-\lambda}^{b} f(t) d t \leq(n-1) \int_{a}^{b} f(t) g(t) d t \leq(n-1) \int_{a}^{a+\lambda} f(t) d t .
$$

The classical Steffensen's inequality (1.1) is thus recaptured.

\section{Applications for Integral Mean}

The following lemma produces alternative identities to those obtained in Lemma 2.1. The current identities involve the integral mean of $f$ over a subinterval $[c, d] \subset[a, b]$.

Lemma 3.1[1] Let $f, g \quad:[a, b] \rightarrow \mathbf{R}$ be integrable functions on $[a, b]$. Define $G(x)=\int_{a}^{x} g(t) d t$ and $\lambda=G(b)=d-c$ where $[c, d] \subset[a, b]$. Then the following identities hold

$\int_{a}^{b} f(x) g(x) d x-\int_{c}^{d} f(y) d y=\lambda[f(b)-\mu(f ; c, d)]-\int_{a}^{b} G(x) d f(x)$

and

$\int_{c}^{d} f(y) d y-\int_{a}^{b} f(x) g(x) d x=\lambda[\mu(f ; c, d)-f(a)]-\int_{a}^{b}[\lambda-G(x)] d f(x)$

where $\mu(f ; c, d)=\frac{1}{d-c} \int_{c}^{d} f(x) d x$ is the integral mean of $f($.$) over [c, d]$.

Theorem 3.1[1] Let $f, g:[a, b] \rightarrow \mathbf{R}$ be integrable functions on $[a, b]$ and let $f$ be nonincreasing. Further, let $g(t) \geq 0$ and $G(x)=\int_{a}^{x} g(t) d t$ with $\lambda=G(b)=d_{i}-c_{i}$ where $\left[c_{i}, d_{i}\right] \subset[a, b]$ for $i=1,2$ and $d_{1} \leq d_{2}$.

(a) Then

$$
\int_{c_{2}}^{d_{2}} f(y) d y-\lambda\left[\mu\left(f ; c_{2}, d_{2}\right)-f(b)\right] \leq \int_{a}^{b} f(x) g(x) d x .
$$

(b) Then

$$
\int_{a}^{b} f(x) g(x) d x \leq \int_{c_{1}}^{d_{1}} f(y) d y+\lambda\left[f(a)-\mu\left(f ; c_{1}, d_{1}\right)\right] .
$$

Remark 3.1 The left side in inequality (3.3) and the right side in inequality (3.4) may be simplified to $\lambda f(b)$ and $\lambda f(a)$ respectively since

$$
\int_{c}^{d} f(y) d y=\lambda \mu(f ; c, d) .
$$

That is, the inequalities (3.3) and (3.4) together become

$$
\lambda f(b) \leq \int_{a}^{b} f(x) g(x) d x \leq \lambda f(a) .
$$

The result should not be overly surprising since it may be obtained directly from the postulates since

$$
\inf _{x \in[a, b]} f(x) \int_{a}^{b} g(x) d x \leq \int_{a}^{b} f(x) g(x) d x \leq \sup _{x \in[a, b]} f(x) \int_{a}^{b} g(x) d x .
$$

The result (3.5) readily follows on noting that 


$$
\int_{a}^{b} g(x)[f(x)-f(b)] d x \geq 0
$$

and

$$
\int_{a}^{b} g(x)[f(a)-f(x)] d x \geq 0 .
$$

The motivation behind Lemma 3.1 and Theorem 3.1 was to obtain a Steffensen like inequality and it was not predictable in advance that the result would reduce to (3.5).

The following theorem provides a new extension to Theorem 3.1 to the case $i=1,2, \ldots, n$.

Theorem 3.2 Let $f, g:[a, b] \rightarrow \mathbf{R}$ be integrable functions on $[a, b]$ and let $f$ be non-increasing. Further, let $g(t) \geq 0$ and $G(x)=\int_{a}^{x} g(t) d t$ with $\lambda=G(b)=d_{i}-c_{i}$ where $\left[c_{i}, d_{i}\right] \subset[a, b]$ for $i=1,2, \ldots, n$ and $d_{1} \leq d_{2} \leq \ldots \leq d_{n}, n=2,3,4, \ldots$.

(a) Then

$$
\begin{aligned}
& \int_{c_{2}}^{d_{2}} f(y) d y+\int_{c_{3}}^{d_{3}} f(y) d y+\ldots+\int_{c_{n}}^{d_{n}} f(y) d y-\lambda\left[\mu\left(f ; c_{2}, d_{2}\right)-f(b)\right] \\
& \quad-\lambda\left[\mu\left(f ; c_{3}, d_{3}\right)-f(b)\right]-\ldots-\lambda\left[\mu\left(f ; c_{n}, d_{n}\right)-f(b)\right] \\
& \leq(n-1) \int_{a}^{b} f(x) g(x) d x .
\end{aligned}
$$

(b) Then

$$
\begin{aligned}
(n-1) & \int_{a}^{b} f(x) g(x) d x \leq \int_{c_{1}}^{d_{1}} f(y) d y+\int_{c_{2}}^{d_{2}} f(y) d y+\ldots+\int_{c_{n-1}}^{d_{n-1}} f(y) d y \\
& +\lambda\left[f(a)-\mu\left(f ; c_{1}, d_{1}\right)\right]+\lambda\left[f(a)-\mu\left(f ; c_{2}, d_{2}\right)\right]+\ldots+ \\
& +\lambda\left[f(a)-\mu\left(f ; c_{n-1}, d_{n-1}\right)\right] .
\end{aligned}
$$

Proof of part (a). Since $f$ is non-increasing and $g(t) \geq 0$ then

Now, from Lemma 4.1, we obtain

$$
-(n-1) \int_{a}^{b} G(x) d f(x) \geq 0 .
$$

$$
\begin{gathered}
U\left(a, b ; c_{2}, d_{2}\right)+U\left(a, b ; c_{3}, d_{3}\right)+\ldots+U\left(a, b ; c_{n}, d_{n}\right)+\lambda\left[\mu\left(f ; c_{2}, d_{2}\right)-f(b)\right] \\
+\lambda\left[\mu\left(f ; c_{3}, d_{3}\right)-f(b)\right]+\ldots+\lambda\left[\mu\left(f ; c_{n}, d_{n}\right)-f(b)\right] \\
=-(n-1) \int_{a}^{b} G(x) d f(x) \geq 0 .
\end{gathered}
$$

Hence

$$
\begin{aligned}
& \int_{a}^{b} f(x) g(x) d x+\int_{a}^{b} f(x) g(x) d x+\ldots+\int_{a}^{b} f(x) g(x) d x-\int_{c_{2}}^{d_{2}} f(y) d y \\
& \quad-\int_{c_{3}}^{d_{3}} f(y) d y-\ldots-\int_{c_{n}}^{d_{n}} f(y) d y+\lambda\left[\mu\left(f ; c_{2}, d_{2}\right)-f(b)\right] \\
& +\lambda\left[\mu\left(f ; c_{3}, d_{3}\right)-f(b)\right]+\ldots+\lambda\left[\mu\left(f ; c_{n}, d_{n}\right)-f(b)\right] \geq 0,
\end{aligned}
$$

and thus (3.6) is valid.

Proof of part (b). Since $f$ is non-increasing and $g(t) \geq 0$ then

$$
-(n-1) \int_{a}^{b}[\lambda-G(x)] d f(x) \geq 0 .
$$

Now, from Lemma 3.1, we obtain

$$
\begin{aligned}
& -U\left(a, b ; c_{1}, d_{1}\right)-U\left(a, b ; c_{2}, d_{2}\right)-\ldots-U\left(a, b ; c_{n-1}, d_{n-1}\right)+\lambda\left[f(a)-\mu\left(f ; c_{1}, d_{1}\right)\right] \\
& \quad+\lambda\left[f(a)-\mu\left(f ; c_{2}, d_{2}\right)\right]+\ldots+\lambda\left[f(a)-\mu\left(f ; c_{n-1}, d_{n-1}\right)\right]
\end{aligned}
$$




$$
=-(n-1) \int_{a}^{b}[\lambda-G(x)] d f(x) \geq 0 .
$$

Hence

$$
\begin{aligned}
& \int_{c_{1}}^{d_{1}} f(y) d y+\int_{c_{2}}^{d_{2}} f(y) d y+\ldots+\int_{c_{n-1}}^{d_{n-1}} f(y) d y-\int_{a}^{b} f(x) g(x) d x \\
& \quad-\int_{a}^{b} f(x) g(x) d x-\ldots-\int_{a}^{b} f(x) g(x) d x+\lambda\left[f(a)-\mu\left(f ; c_{1}, d_{1}\right)\right] \\
& +\lambda\left[f(a)-\mu\left(f ; c_{2}, d_{2}\right)\right]+\ldots+\lambda\left[f(a)-\mu\left(f ; c_{n-1}, d_{n-1}\right)\right] \geq 0,
\end{aligned}
$$

giving (3.7).

Remark 3.2 The left side in inequality (3.6) and the right side in inequality (3.7) may be simplified to $(n-1) \lambda f(b)$ and $(n-1) \lambda f(a)$ respectively since

$$
\int_{c}^{d} f(y) d y=\lambda \mu(f ; c, d) .
$$

That is,

the inequalities (3.6) and (3.7) together become

$$
(n-1) \lambda f(b) \leq(n-1) \int_{a}^{b} f(x) g(x) d x \leq(n-1) \lambda f(a)
$$

which is (3.5).

\section{Conclusion}

In the context of this paper, we presented generalizations of Steffensen's inequality given by Cerone. We obtained some new results regarding Cerone's generalizations. Also we introduced new applications for integral mean.

For future work, we recommend to find special functions that achieve the conditions of Steffensen's inequality and get new formats.

\section{References}

[1] P. Cerone, (2001). On some generalizations of Steffensen's inequality and related results. J. Ineq. Pure and Appl. Math, 2(3).

[2] J.Jakšetić, J. Pečarić, and K.Smoljak,(2015). Extension of Cerone's generalizations of Steffensen's inequality. Jordan Journal of Mathematics and Statistics, 8 (2), 179-194 .

[3] D. S.Mitrinovic , \& P. M. Vasic, (1970). Analytic inequalities (Vol. 1). Berlin: Springer-verlag.

[4] B. G. Pachpatte, (2005). Mathematical inequalities (Vol. 67). Elsevier.

[5] J.Pečarić, A. Perušić , \& K. Smoljak, (2014). Cerone’s generalizations of Steffensen's inequality. Tatra mountains mathematical publications, 58(1), 53-75.

[6] J. F. Steffensen, (1918). On certain inequalities between mean values, and their application to actuarial problems. Scandinavian Actuarial Journal, 1918(1), 82-97. 